\title{
First Passage Time of a Markov Chain That Converges to Bessel Process
}

\author{
Moussa Kounta \\ Mathematics Department, University of the Bahamas, Nassau, Bahamas \\ Correspondence should be addressed to Moussa Kounta; moussakounta@gmail.com
}

Received 16 March 2017; Accepted 27 August 2017; Published 3 December 2017

Academic Editor: Tongxing Li

Copyright (C) 2017 Moussa Kounta. This is an open access article distributed under the Creative Commons Attribution License, which permits unrestricted use, distribution, and reproduction in any medium, provided the original work is properly cited.

We investigate the probability of the first hitting time of some discrete Markov chain that converges weakly to the Bessel process. Both the probability that the chain will hit a given boundary before the other and the average number of transitions are computed explicitly. Furthermore, we show that the quantities that we obtained tend (with the Euclidian metric) to the corresponding ones for the Bessel process.

\section{Introduction}

The study of the probability of the hitting times for stochastic differential equations is an active area of research and they are of great interest in many applications, for example, in finance, the study of path dependent exotic options as barrier options, in percolation theory [1], in optimal control problems [2], and in neuroscience [3]. In this paper we investigate the discrete version of Bessel processes defined by an stochastic differential equation. It is well known [4] that, given a diffusion process defined by a stochastic differential equation, we can produce a discrete Markov chain that converges weakly to the solution of this stochastic differential equation (by making use of a binomial approximation). In this paper we show that the probability of the first passage times and the number of the transitions of this discrete Markov chain tend to the corresponding ones for the continuous times Bessel process.

The discrete versions of stochastic processes are interesting in themselves; for instance, in quantum mechanics the motion of a particle should be essentially discontinuous and random. Moreover, in [5] the authors show the application of the discrete version of Cox-Ingersoll-Ross process in hydrology.

In this paper, we consider the so-called gambler's ruin problem for a discrete-time Markov chain that converges to a Bessel process. Phenomena governed by Bessel processes abound in the physical world, as in the case of growth phenomena governed by Stochastic Loewner Evolution (SLE) [1]. Other phenomena include first hitting time of Bessel processes in the study of systems at or near the point of phase transition in statistical physics. In finance, a typical example is the study of stock price. It is well known that in a volatilitystabilized market the stock prices can be represented in terms of Bessel processes. Since the stock price does not vary completely continuously, the discrete formulas that will be derived in the present paper would be interesting.

The paper is organized as follows. In Section 2, we briefly describe the transition probability derived from the Bessel stochastic differential equation. Our main contribution is in the third section (Section 3.2): We find the explicit formula of the average number of transitions needed to end the game that was impossible to obtain in [6]. We also show the sequence of the probability of the first passage times and the average number of transitions to end the game converges (with Euclidean metric) to the corresponding values in the continuous case.

\section{Bessel Process Defined by a Stochastic Differential Equation and Simple Binomial Approximation}

2.1. Preliminaries on Bessel Processes. We consider the Bessel process defined by the following differential equation:

$$
\begin{array}{r}
d R(t)=\left(v+\frac{1}{2}\right) \frac{1}{R(t)} d t+d B(t), \\
\text { where } B(t) \text { Brownian motion. }
\end{array}
$$


Next, let $a=v+1 / 2$ and if there is no ambiguity we remove the index $t$ on $R$. Assume that $R(0)=r \in(1, N)$, where $N \in \mathbb{N}$ (for simplicity), and define

$$
\tau(r)=\inf \{t>0: R(t)=1 \text { or } N \mid R(0)=r\} .
$$

As is well known (see, e.g., [7], page 220), the probability

$$
p(r)=P[R[\tau(r)]=N] .
$$

satisfies the ordinary differential equation:

$$
\frac{1}{2} p^{\prime \prime}(r)+\frac{a}{r} p^{\prime}(r)=0, \quad \text { with } p(1)=0, p(N)=1 .
$$

We easily find that, if $a \neq 1 / 2$

$$
p(r)=\frac{r^{1-2 a}-N^{1-2 a}}{N^{1-2 a}-1} \text { for } 1 \leqslant r \leqslant N,
$$

and when $a=1 / 2$, the solutions is

$$
p(r)=\frac{\ln r}{\ln N} \text { for } 1 \leqslant r \leqslant N .
$$

Let

$$
m(u)=E[\tau(u)]
$$

In [7], we see the function $m(u)$ satisfies the second-order ordinary differential equation:

$$
\frac{1}{2} m^{\prime \prime}(u)+\frac{a}{u} m^{\prime}(u)=-1 \quad \text { with } m(1)=m(N)=0 .
$$

The general solution of this equation is

$$
m(u)=\int_{1}^{u} r^{-2 a}\left(-2 \int_{r}^{N} y^{2 a} d y+c_{0}\right) d r,
$$

where

$$
c_{0}=\frac{2 \int_{1}^{N} r^{-2 a}\left(\int_{r}^{N} y^{2 a} d y\right) d r}{\int_{1}^{N} r^{-2 a} d r}, \quad a \neq \frac{1}{2}
$$

2.2. Preliminaries: Binomial Approximation. In this section we recall binomial approximation briefly; for more details please see [4]. We wish to find a sequence of stochastic processes that converges in distribution to process (1) over the time interval $[0, T]$.

Take the interval $[0, T]$, and chop it into $n$ equal pieces $\left\{0, t_{1}, t_{2}, \ldots, t_{n}\right\}$ of length $\Delta t \equiv T / n, t_{j}=j \Delta t$. Define a sequence $R_{t_{j}}=1+j \Delta r$ of binomial approximations from (1), which is constant between nodes, such that at any given node $R_{t_{j}}$ the process jumps up to $R_{t_{j}}^{+}=R_{t_{j+1}}$ (resp., down to $R_{t_{j}}^{-}=R_{t_{j-1}}$ ) with probability $p_{r_{t_{j}}, r_{t_{j+1}}}$ (resp., $p_{r_{t_{j}}, r_{t_{j-1}}}$ ), and stays at the node with probability $1-p_{r_{t_{j}}, r_{t_{j+1}}}-p_{r_{t_{j}}, r_{t_{j-1}}}$.

$$
\begin{aligned}
& \mathbf{P}\left[R_{t_{j+1}}=R^{+}\left(r_{t_{j}}\right) \mid R_{t_{j}}=r_{t_{j}}\right]=p_{r_{t_{j}}, r_{t_{j+1}}}, \\
& \mathbf{P}\left[R_{t_{j+1}}=R^{-}\left(r_{t_{j}}\right) \mid R_{t_{j}}=r_{t_{j}}\right]=p_{r_{t_{j}}, r_{t_{j-1}},}, \\
& \mathbf{P}\left[R_{t_{j+1}}=R_{t_{j}}=c \mid R_{t_{j}}=r_{t_{j}}\right]=1-p_{r_{t_{j}}, r_{t_{j-1}}}-p_{r_{t_{j}}, r_{t_{j+1}}} .
\end{aligned}
$$

The local drift of $R_{t_{j}}$ is given by

$$
\mu_{\Delta t}=\left\{p_{r_{t_{j}}, r_{t_{j+1}}}-p_{r_{t_{j}}, r_{t_{j-1}}}\right\} \Delta r
$$

and the local second moment of $R_{t_{j}}$ is given by

$$
\begin{aligned}
& \sigma_{\Delta t} \\
& \quad=\left[p_{r_{t_{j}}, r_{t_{j+1}}}+p_{r_{t_{j}}, r_{t_{j-1}}}-\left\{p_{r_{t_{j}}, r_{t_{j+1}}}-p_{r_{t_{j}}, r_{t_{j-1}}}\right\}^{2}\right](\Delta r)^{2} .
\end{aligned}
$$

On the other hand,

$$
\begin{gathered}
\lim _{\Delta t \rightarrow 0} \frac{\mathbf{E}\left[\left\{R_{t_{j+1}}-R_{t_{j}}\right\} \mid R_{t_{j}}\right]}{\Delta t}=\lim _{\Delta r, \Delta t \rightarrow 0} \frac{\mu_{\Delta t}}{\Delta t}=\frac{a}{R_{t_{j}}}, \\
\lim _{\Delta t \rightarrow 0} \frac{\operatorname{var}\left[\left\{R_{t_{j+1}}-R_{t_{j}}\right\} \mid R_{t_{j}}\right]}{\Delta t}=\lim _{\Delta r, \Delta t \rightarrow 0} \frac{\sigma_{\Delta t}}{\Delta t}=1 .
\end{gathered}
$$

By solving (12), (13), and (14), we obtain

$$
\begin{aligned}
& p_{r_{t_{j}}, r_{t_{j+1}}}=\frac{1}{2 A}\left[1+\frac{a}{r_{t_{j}}} \Delta r+\Delta t\left(\frac{a}{r_{t_{j}}}\right)^{2}\right], \\
& p_{r_{t_{j}}, r_{t_{j-1}}}=\frac{1}{2 A}\left[1-\frac{a}{r_{t_{j}}} \Delta r+\Delta t\left(\frac{a}{r_{t_{j}}}\right)^{2}\right] .
\end{aligned}
$$

Since $1 / r_{t_{j}}$ is bounded, we can write $\Delta t\left(a / r_{t_{j}}\right)^{2}=O(\Delta t)$; hence we obtain

$$
\begin{aligned}
& p_{r_{t_{j}}, r_{t_{j+1}}}=\frac{1}{2 A}\left[1+\frac{a \Delta r}{r_{t_{j}}}+O(\Delta t)\right], \\
& p_{r_{t_{j}}, r_{t_{j-1}}}=\frac{1}{2 A}\left[1-\frac{a \Delta r}{r_{t_{j}}}+O(\Delta t)\right] .
\end{aligned}
$$

We obtain the following transition probabilities $p_{r_{t_{j}}, r_{t_{j+1}}}$, $p_{r_{t_{j}}, r_{t_{j-1}}}$, and $p_{r_{t_{j}}, r_{t_{j}}}$ given by

$$
\begin{aligned}
p_{r_{t_{j}}, r_{t_{j+1}}} & \simeq \frac{1}{2 A}\left[1+\frac{a \Delta r}{r_{t_{j}}}\right], \\
p_{r_{t_{j}}, r_{t_{j-1}}} & \simeq \frac{1}{2 A}\left[1-\frac{a \Delta r}{r_{t_{j}}}\right], \\
p_{r_{t_{j}}, r_{t_{j}}} & \simeq 1-\frac{1}{A} .
\end{aligned}
$$

We state the following assumptions, under which $R_{t_{j}}$ converges weakly to $R_{t}$ (see [8]).

Assumption 1. With probability 1, a solution $\left\{R_{t}\right\}$ of the stochastic integral equation

$$
R_{t}=R_{0}+\int_{0}^{t} \frac{a}{R(s)} d s+\int_{0}^{t} d B_{s}
$$

exists for $0<t<\infty$, and is distributionally unique. 
Assumption 2. For all $\delta>0$ and all $T>0$,

$$
\begin{aligned}
& \lim _{\Delta t \rightarrow 0} \sup _{\substack{|r| \leqslant \delta \\
0 \leqslant t \leqslant T}}\left|R_{\Delta t}^{+}(r, t)-r(t)\right|=0, \\
& \lim _{\Delta t \rightarrow 0} \sup _{\substack{|r| \leqslant \delta \\
0 \leqslant t \leqslant T}}\left|R_{\Delta t}^{-}(r, t)-r(t)\right|=0 .
\end{aligned}
$$

Assumption 3. For all $\delta>0$ and all $T>0$,

$$
\begin{array}{r}
\lim _{\Delta t \rightarrow 0} \sup _{\substack{|r| \leqslant \delta \\
0 \leqslant t \leqslant T}}\left|\mu_{\Delta t}(r, t)-\frac{a}{r(t)}\right|=0, \\
\lim _{\Delta t \rightarrow 0} \sup _{\substack{|r| \leqslant \delta \\
0 \leqslant t \leqslant T}}\left|\sigma_{\Delta t}(r, t)-1\right|=0 .
\end{array}
$$

Theorem 4. Under Assumptions 1-3, $R_{t_{j}} \Rightarrow R_{t}$ converge to the solution of (1), where $\Rightarrow$ denotes weak convergence.

Next, we assume $R_{t_{j}}$ is bounded for any $j=0,1, \ldots, n$.

\section{Discrete Value of the Probability and the Average Number of Transitions of First Passage Time, of the Bessel Process}

Let

$$
\begin{aligned}
& T_{j}=\inf \left\{m>0: R_{t_{m}}=1 \text { or } N \mid R_{0}=1+j \Delta r\right\}, \\
& p_{j}=P\left[R_{T_{j}}=N\right] \\
& d_{j}=E\left[R_{T_{j}}=N\right] .
\end{aligned}
$$

In this section, we will compute the quantity $p_{j}$ for $j \in$ $\{1, \ldots, k-1\}$. We will show that $p_{j}$ converges to the function $p(r)$ for the Bessel process as $\Delta r$ decreases to zero and $k$ tends to infinity in such a way that $1+k \Delta r$ remains equal to $N$.

\subsection{Computation of the Probability $p_{j}$}

3.1.1. Assuming First That $\Delta r=1$. Then the state space is $\{1,2, \ldots, N\}$ and the transition probabilities become

$$
\begin{aligned}
p_{j, j+1} & =\frac{1}{2 A}\left\{1+\frac{a}{j}\right\}, \\
p_{j, j-1} & =\frac{1}{2 A}\left\{1-\frac{a}{j}\right\}, \\
p_{j, j} & =1-\frac{1}{A},
\end{aligned}
$$

for $j \in\{2, \ldots, N-1\}$. It is well known that the probability defined in (22) satisfies the following difference equation:

$$
p_{j}=p_{j, j+1} p_{j+1}+p_{j, j-1} p_{j-1}+p_{j, j} p_{j} .
$$

Equation (24) can be rewritten as

$$
w_{j}-\left(\frac{j-a}{j+a}\right) w_{j-1}=0 \quad \text { where } w_{j}=p_{j}-p_{j-1}
$$

We find that the solution of this first-order difference equation that satisfies the boundary condition $w_{1}=p_{2}$ is given by

$$
\begin{aligned}
& w_{j}=f(a) \frac{\Gamma(j+1-a)}{\Gamma(j+1+a)}, \\
& \text { where } f(a)=-\frac{(1+a) \Gamma(a)}{(1-a) \Gamma(-a)} .
\end{aligned}
$$

We have the following lemma.

Lemma 5. For $a \neq 1 / 2$, the unique solution of (24) subject to the boundary conditions (4) is given by

$$
p_{j}=\frac{(j+a)(\Gamma(j+1-a) / \Gamma(j+1+a))-(1+a)(\Gamma(2-a) / \Gamma(2+a))}{(N+a)(\Gamma(N+1-a) / \Gamma(N+1+a))-(1+a)(\Gamma(2-a) / \Gamma(2+a))} \quad \text { for } j=1,2, \ldots, N
$$

Proof. We have

$$
\begin{aligned}
p_{j+1}-p_{j} & =f(c) \frac{\Gamma(j+1-c)}{\Gamma(j+1+c)} \\
& =\frac{f(c)}{\Gamma(2 c)} \int_{0}^{1} t^{j} t^{-c}(1-t)^{2 c-1} d t, \\
p_{j}-p_{1} & =\sum_{k=1}^{j-1}\left(p_{k+1}-p_{k}\right) \\
& =\frac{f(c)}{\Gamma(2 c)} \int_{0}^{1} \frac{t-t^{j}}{1-t} t^{-c}(1-t)^{2 c-1} d t .
\end{aligned}
$$

Since $p_{1}=0$ and $B(z, w)=\int_{0}^{1} t^{z-1}(1-t)^{w-1} d t=\int_{0}^{\infty}\left(t^{z-1} /(1+\right.$ $\left.t)^{z+w}\right) d t=\Gamma(z) \Gamma(w) / \Gamma(z+w)$ we obtain,

$$
\begin{aligned}
p_{j} & =\frac{f(c)}{\Gamma(2 c)} \int_{0}^{1} t^{1-c}(1-t)^{2 c-2} d t-\frac{f(c)}{\Gamma(2 c)} \\
& \cdot \int_{0}^{1} t^{j-c}(1-t)^{2 c-2} d t \\
& =\frac{f(c) \Gamma(2 c-1)}{\Gamma(2 c)}\left[\frac{(c+1) \Gamma(2-c)}{\Gamma(2+c)}\right. \\
& \left.-\frac{(j+c) \Gamma(j+1-c)}{\Gamma(j+c+1)}\right] .
\end{aligned}
$$


By applying boundary conditions (4), we obtain

$$
\begin{aligned}
1 & =p_{N}=\frac{f(a) \Gamma(2 a-1)}{\Gamma(2 a)}\left[\frac{(a+1) \Gamma(2-a)}{\Gamma(2+a)}-\frac{(N+a) \Gamma(N+1-a)}{\Gamma(N+a+1)}\right], \\
\frac{f(a) \Gamma(2 a-1)}{\Gamma(2 a)} & =\frac{1}{(a+1) \Gamma(2-a) / \Gamma(2+a)-(N+a) \Gamma(N+1-a) / \Gamma(N+a+1)},
\end{aligned}
$$

and hence

$$
p_{j}=\frac{(j+a)(\Gamma(j+1-a) / \Gamma(j+1+a))-(1+a)(\Gamma(2-a) / \Gamma(2+a))}{(N+a)(\Gamma(N+1-a) / \Gamma(N+1+a))-(1+a)(\Gamma(2-a) / \Gamma(2+a))} \quad \text { for } j=1,2, \ldots, N
$$

Now we suppose $a \neq 1 / 2$.

Proof. Indeed, we have

Lemma 6. When a tends to $1 / 2$, the solution becomes

$$
p_{j}=\frac{\Psi(j+1 / 2)-2+\gamma+2 \ln 2}{\Psi(N+1 / 2)-2+\gamma+2 \ln 2},
$$

where $\Psi(z)=\Gamma^{\prime}(z) / \Gamma(z)$ and $\gamma$ is Euler's constant.

$$
\begin{aligned}
p_{j}= & \frac{(j+a)(\Gamma(j+1-a) / \Gamma(j+1+a))-(1+a)(\Gamma(2-a) / \Gamma(2+a))}{(N+a)(\Gamma(N+1-a) / \Gamma(N+1+a))-(1+a)(\Gamma(2-a) / \Gamma(2+a))} \\
= & \frac{(j+a)(\Gamma(j+1-a) /(j+a) \Gamma(j+a))-(1+a) \Gamma(2-a) /(1+a) \Gamma(1+a)}{(N+a) \Gamma(N+1-a) /(N+a) \Gamma(N+a)-(1+a) \Gamma(2-a) /(1+a) \Gamma(1+a)} \text { for } j=1,2, \ldots, N, \\
\lim _{a \rightarrow 1 / 2} p_{j}= & \lim _{a \rightarrow 1 / 2} \frac{(d / d a)(\Gamma(j+1-a) / \Gamma(j+a))-(d / d a)(\Gamma(2-a) / \Gamma(1+a))}{(d / \Gamma(N+1-a) / \Gamma(N+a))-(d / d a)(\Gamma(2-a) / \Gamma(1+a))}=\frac{\Psi(j+1 / 2)-\Psi(3 / 2)}{\Psi(N+1 / 2)-\Psi(3 / 2)}
\end{aligned}
$$

Since $\Psi(3 / 2)=2-\gamma-2 \ln 2$ (see [9]), we obtain

$$
\lim _{a \rightarrow 1 / 2} p_{j}=\frac{\Psi(j+1 / 2)-2+\gamma+2 \ln 2}{\Psi(N+1 / 2)-2+\gamma+2 \ln 2}
$$

3.1.2. Now, in the General Case When $\Delta r>0$. We must solve the difference equation:

$$
\begin{aligned}
p_{j}= & \frac{1}{2 A}\left\{1+\frac{a \Delta r}{1+j \Delta r}\right\} p_{j+1} \\
& +\frac{1}{2 A}\left\{1-\frac{a \Delta r}{1+j \Delta r}\right\} p_{j-1}+\left\{1-\frac{1}{A}\right\} p_{j},
\end{aligned}
$$

which can be simplified to

$$
p_{j+1}-p_{j}=\frac{1-a \Delta r /(1+j \Delta r)}{1+a \Delta r /(1+j \Delta r)}\left(p_{j}-p_{j-1}\right) .
$$

The boundary conditions become

$$
\begin{aligned}
& p_{0}=0, \\
& p_{k}=1 .
\end{aligned}
$$

Next, proceeding as above, we obtain that if $a \neq 1 / 2$, the probability $p_{j}$ is given by 


$$
\begin{aligned}
& p_{j} \\
& =\frac{(1+j \Delta r+a \Delta r)(\Gamma((1+(j+1) \Delta r-a \Delta r) / \Delta r) / \Gamma((1+(j+1) \Delta r+a \Delta r) / \Delta r))-(1+a \Delta r)(\Gamma((1+\Delta r-a \Delta r) / \Delta r) / \Gamma((1+\Delta r+a \Delta r) / \Delta r))}{(1+k \Delta r+a \Delta r)(\Gamma((1+(k+1) \Delta r-a \Delta r) / \Delta r) / \Gamma((1+(k+1) \Delta r+a \Delta r) / \Delta r))-(1+a \Delta r)(\Gamma((1+\Delta r-a \Delta r) / \Delta r) / \Gamma((1+\Delta r+a \Delta r) / \Delta r))} .
\end{aligned}
$$

Writing

$$
\begin{gathered}
n=1+j \Delta r, \\
N=1+k \Delta r,
\end{gathered}
$$

in terms of $n$ and $N$, this expression becomes

$$
\begin{aligned}
& p_{n} \\
& =\frac{(n+a \Delta r)(\Gamma((n+\Delta r-a \Delta r) / \Delta r) / \Gamma((n+\Delta r+a \Delta r) / \Delta r))-(1+a \Delta r)(\Gamma((1+\Delta r-a \Delta r) / \Delta r) / \Gamma((1+\Delta r+a \Delta r) / \Delta r))}{(N+a \Delta r)(\Gamma((N+\Delta r-a \Delta r) / \Delta r) / \Gamma((N+\Delta r+a \Delta r) / \Delta r))-(1+a \Delta r)(\Gamma((1+\Delta r-a \Delta r) / \Delta r) / \Gamma((1+\Delta r+a \Delta r) / \Delta r))},
\end{aligned}
$$

for $n \in\{1,1+\Delta r, \ldots, 1+k \Delta r=N\}$. The solution reduces to

$$
\begin{aligned}
p_{n}=\frac{\Psi((2 n+\Delta r) / 2 \Delta r)-\Psi((2+\Delta r) / 2 \Delta r)}{\Psi((2 N+\Delta r) / 2 \Delta r)-\Psi((2+\Delta r) / 2 \Delta r)} & \\
& \text { if } a=\frac{1}{2} .
\end{aligned}
$$

We can now state the following proposition.

Proposition 7. Let $n=1+j \Delta r$ for $j \in\{0,1, \ldots, k\}$, with $k$ such that $1+k \Delta r=N$. The probability $p_{n}$ that the discrete-time Markov chain defined in Section 2.1, starting from $n$, will hit $N$ before 1 is given by (40) if $a \neq 1 / 2$. The value of the probability $p_{n}$ tends to the function in (41) when a tends to $1 / 2$.

Next, when $a=1 / 2$, by making use of the following approximation $\Psi(z) \sim \ln z$ for large $z$, we can write that

$$
\begin{aligned}
& \lim _{\Delta r \downarrow 0} p_{n}=\lim _{\Delta r \downarrow 0} \frac{\ln (2 n+\Delta r)-\ln (2+\Delta r)}{\ln (2 N+\Delta r)-\ln (2+\Delta r)}=\frac{\ln n}{\ln N} \\
& \qquad \text { for } n \in[1, N] .
\end{aligned}
$$

This expression corresponds to the function $p(x)$ given in (6), obtained when $a=1 / 2$. Finally, we have

$$
\frac{\Gamma(z+\alpha)}{\Gamma(z+\beta)} \propto z^{\alpha-\beta}\left(1+O\left(\frac{1}{z}\right)\right)
$$

as $|z|$ tends to infinity (if $|\arg (z+\alpha)|<\pi$; see [9]). Hence, in the case $a \neq 0,1 / 2$, we can write that

$$
\lim _{\Delta r \downarrow 0} p_{n}=\frac{n^{1-2 a}-1}{N^{1-2 a}-1}, \quad \text { for } 1 \leqslant n \leqslant N .
$$

Therefore, we retrieve the formula for $p(r)$ in (5). In the next section, we will derive the formulas that correspond to the function $m(r)$ in Section 1.

3.2. Computation of the Mean Number of Transitions $d_{j}$ Needed to End the Game. We now turn to the problem of computing the mean number $d_{j}$ of transitions that the Markov chain $\left\{R_{t_{m}}, m=0,1, \ldots\right\}$, starting from $R_{0}=1+j \Delta r$, takes to reach either 1 or $1+k \Delta r=N$. Unlike the results of [6], we find the explicit formula of the average number of transitions needed to end the game.

3.2.1. The Case $\Delta r=1$. If $\Delta r=1$, the state of the Markov chain is the set $\{1,2, \ldots, N\}$. Conditioning on the result of the first transition of the chain and taking this first transition into account, we obtain that the quantity $d_{j}=E\left[R_{T_{j}}=N\right]$ satisfies the second-order linear, nonhomogeneous difference equation

$$
\begin{aligned}
& d_{j}=p_{j, j+1} d_{j+1}+p_{j, j-1} d_{j-1}+p_{j, j} d_{j}+1 \\
& \qquad \text { for } j=2, \ldots, N-1,
\end{aligned}
$$

where the probabilities $p_{j, j+1}, p_{j, j-1}$, and $p_{j, j}$ are defined, respectively, in (17), with the boundary conditions:

$$
d_{1}=d_{N}=0
$$

We have

$$
\begin{aligned}
& q_{j}-\left(\frac{j-a}{j+a}\right) q_{j-1}=-2 A \frac{j}{j+a} \\
& \text { where } q_{j}=d_{j+1}-d_{j} .
\end{aligned}
$$

Let $r_{1}(j)=(j-a) /(j+a), r_{2}(j)=-2 A(j /(j+a))$.

Now, by making use of the formula of Batchelder [10] (5, p.62), we obtain that a formal particular rational solution $R_{j}^{*}$ of (47) is given by

$$
\begin{gathered}
R_{j}^{*}=-\sum_{k=0}^{\infty} \frac{r_{2}(j+k)}{\prod_{i=0}^{k} r_{1}(j+i)}=2 A \frac{\Gamma(j-a)}{\Gamma(j+a)} \\
\cdot \sum_{k=0}^{\infty} \frac{(j+k) \Gamma(j+a+k)}{(j+k-a) \Gamma(j-a+k)}=2 A
\end{gathered}
$$




$$
\begin{aligned}
& \frac{\Gamma(j-a)}{\Gamma(j+a)}\left[\sum_{k=0}^{\infty} \frac{\Gamma(j+a+k+1)}{\Gamma(j-a+k+1)}\right. \\
& \left.-a \sum_{k=0}^{\infty} \frac{\Gamma(j+a+k)}{\Gamma(j-a+k+1)}\right] .
\end{aligned}
$$

Then

$$
\begin{aligned}
R_{j}^{*} & =2 A \frac{\Gamma(j-a)}{\Gamma(j+a)}\left[\frac{\Gamma(j+a+1)}{\Gamma(j-a+1)} \Gamma(1)\right. \\
& \cdot F(j+a+1,1 ; j-a+1,1)-a \frac{\Gamma(j+a) \Gamma(1)}{\Gamma(j-a)} \\
& \cdot F(j+a, 1 ; j-1,1)]
\end{aligned}
$$

where $F(a, b ; c ; z)=(\Gamma(c) / \Gamma(a) \Gamma(b)) \sum_{n=0}^{\infty}(\Gamma(a+n) \Gamma(b+$ $n) / \Gamma(c+n))\left(z^{n} / n !\right)$, hypergeometric function.

Proposition 8. In the case $\Delta r=1$, the mean number $d_{j}$ of transitions needed by the Markov chain, starting from $j$, to reach either 1 or $N$ can be expressed as follows:

$$
d_{j}=\sum_{k=1}^{j-1}\left\{\alpha_{0} \frac{\Gamma(k+1-a)}{\Gamma(k+1+a)}+R_{k}^{*}\right\},
$$

for $j=1, \ldots, N$, where the constant $\alpha_{0}$ is given by

$$
\alpha_{0}=\frac{-\sum_{k=1}^{N-1} R_{k}^{*}}{\sum_{k=1}^{N-1}(\Gamma(k+1-a) / \Gamma(k+1+a))} .
$$

3.2.2. The Case $\Delta r>0$. To obtain the solution to our problem in the case when $\Delta r>0$, we must find a particular solution of

$$
\begin{aligned}
d_{j} & =\frac{1}{2 A}\left\{1+\frac{a \Delta r}{1+j \Delta r}\right\} d_{j+1} \\
& +\frac{1}{2 A}\left\{1-\frac{a \Delta r}{1+j \Delta r}\right\} d_{j-1} \\
+ & \left\{1+\frac{1}{A}\right\} d_{j}+1,
\end{aligned}
$$

for $j=1, \ldots, k-1$, with $d_{0}=d_{k}=0$; proceeding as above by letting $d_{j+1}-d_{j}=q_{j}$, we can rewrite this second-order linear, nonhomogeneous difference equation as follows:

$$
q_{j+1}-\left(\frac{1+j \Delta r-a \Delta r}{1+j \Delta r+a \Delta r}\right) q_{j}=-2 A \frac{(1+j \Delta r)}{1+j \Delta r+a \Delta r} .
$$

We deduce from the preceding subsection that

$$
\begin{aligned}
R_{j}^{*}= & -2 A \frac{\Gamma(1 / \Delta r+j-a)}{\Gamma(1 / \Delta r+j+a)} \\
& \cdot \sum_{n=0}^{\infty}\left(\frac{1}{\Delta r}+n+k\right) \frac{\Gamma(1 / \Delta r+k+n+a)}{\Gamma(1 / \Delta r+k+n-a+1)} .
\end{aligned}
$$

To complete the work, we now show that $d_{j} \times \Delta t$ converges to the function $m(r)$ when we choose $\Delta t=(\Delta r)^{2} / A$. To do so, we express the general solution of (53) in terms of $r=1+j \Delta r$ and write $d_{j}$ as $d(r)$ :

$$
\begin{gathered}
d^{\prime}(r) \simeq \alpha_{0}(\Delta r) \frac{\Gamma(r / \Delta r-a+1) \Gamma(1 / \Delta r+a+1)}{\Gamma(1 / \Delta r+a+1) \Gamma(r / \Delta r-a+1)} \\
-2 A \frac{\Gamma(r / \Delta r-a)}{\Gamma(r / \Delta r+a)} \\
\quad \sum_{n=0}^{\infty} \frac{((r+n \Delta r) / \Delta r) \Gamma((r+n \Delta r) / \Delta r+a)}{\Gamma((r+n \Delta r) / \Delta r-a+1)}
\end{gathered}
$$

since for $|z| \rightarrow \infty$ and $|\arg (z+a)|<\pi$ (see [9])

$$
\frac{\Gamma(z+a)}{\Gamma(z+b)} \simeq z^{a-b}\left(1+O\left(\frac{1}{z}\right)\right) .
$$

Then for $\Delta r$ small enough, we may write that

$$
\begin{aligned}
& d^{\prime}(r) \simeq \alpha_{0}(\Delta r) r^{-2 a}\left(1+O\left(\frac{\Delta r}{r}\right)\right)\left(\frac{1}{\Delta r}\right)^{-2 a} \times(1 \\
& +O(\Delta r))-2 A\left(\frac{r}{\Delta r}\right)^{-2 a}\left(1+O\left(\frac{\Delta r}{r}\right)\right) \\
& \quad \times \sum_{n=0}^{\infty}\left(\frac{1}{\Delta r}\right)(r+n \Delta r)\left(\frac{r+n \Delta r}{\Delta r}\right)^{2 a-1} \\
& \cdot\left(1+O\left(\frac{\Delta r}{r}\right)\right) .
\end{aligned}
$$

Next, let

$$
\begin{aligned}
& S(r) \\
& =\sum_{n=0}^{\infty}\left(\frac{1}{\Delta r}\right)(r+n \Delta r)\left(\frac{r+n \Delta r}{\Delta r}\right)^{2 a-1}\left(1+O\left(\frac{\Delta r}{r}\right)\right) \\
& =\left(1+O\left(\frac{\Delta r}{r}\right)\right)\left(\frac{1}{\Delta r}\right)^{2 a+1} \sum_{n=0}^{\infty}(r+n \Delta r)^{2 a} \Delta r .
\end{aligned}
$$

Now, let $l=[(N-r) / \Delta r]$, where $[x]$ is the least integer greater than or equal to $x$. Then, for small $\Delta r$, we may write that

$$
\begin{aligned}
& S(r) \\
& =\lim _{l \rightarrow \infty}\left(1+O\left(\frac{\Delta r}{r}\right)\right)\left(\frac{1}{\Delta r}\right)^{2 a+1} \sum_{n=0}^{l}(r+n \Delta r)^{2 a} \Delta r \\
& =\left(1+O\left(\frac{\Delta r}{r}\right)\right)\left(\frac{1}{\Delta r}\right)^{2 a+1} \int_{r}^{N} y^{2 a} d y .
\end{aligned}
$$

Thus, from what precedes, we deduce that

$$
\begin{aligned}
\lim _{\Delta r \rightarrow 0} d^{\prime}(r) \frac{(\Delta r)^{2}}{2 A}= & \alpha_{0}(\Delta r)\left(\frac{\Delta r}{2 A}\right) r^{-2 a} \\
& -2 r^{-2 a} \int_{r}^{N} y^{2 a} d y
\end{aligned}
$$


and we get

$$
\lim _{\Delta r \rightarrow 0} d^{\prime}(r) \Delta t=r^{-2 a} \frac{\alpha_{0}(\Delta r)}{\Delta r} \Delta t-2 r^{-2 a} \int_{r}^{N} y^{2 a} d y .
$$

Hence, if we let $D(u)=\lim _{\Delta r \rightarrow 0} d(u) \Delta t$, we can write (by making use of the boundary condition $D(1)=0$ ) that

$$
\begin{aligned}
& D(u) \\
& \quad=\int_{1}^{u}\left\{r^{-2 a} \lim _{\Delta r \rightarrow 0} \frac{\alpha_{0}(\Delta r)}{\Delta r} \Delta t-2 r^{-2 a} \int_{r}^{N} y^{2 a} d y\right\} d r .
\end{aligned}
$$

Finally, to satisfy the boundary condition $D(N)=0$, we find that the constant $\alpha_{0}(\Delta r)$ must be chosen so that $D(u)=m(u)$. That is, we retrieve formula (9) for the function $m(u)$.

\section{Conclusion and Future Research}

As it is well known, the Bessel process is a very important model in financial mathematics. In practice, stock or commodity prices vary discretely over time. Therefore, it is interesting to derive formulas for $p_{j}$ and $d_{j}$ for Markov chains that are as close as we want to the diffusion process. Next, we will try to extend the result in the case of twodimensional diffusion process, which had a lot of applications in real life portfolio insurance [11, 12] and hydrology [13]. It is a difficult task, since we have to solve some systems of difference equation in two variables.

\section{Conflicts of Interest}

The author declares that there are no conflicts of interest regarding the publication of this paper.

\section{Acknowledgments}

The authors acknowledge the University of the Bahamas Internal Grants Program for Research, Creative and Artistic Proposals (2016-2017) for generously supporting this project.

\section{References}

[1] G. F. Lawler, "Conformal invariance and 2D statistical physics," American Mathematical Society. Bulletin. New Series, vol. 46, no. 1, pp. 35-54, 2009.

[2] A. Vollert, A Stochastic Control Framework for Real Options in Strategic Evaluation, Birkhäuser Boston, Inc., Boston, MA, 2003.

[3] H. C. Tuckwell, Stochastic processes in the neurosciences, vol. 56 of CBMS-NSF Regional Conference Series in Applied Mathematics, Society for Industrial and Applied Mathematics (SIAM), Philadelphia, PA, 1989.

[4] D. B. Nelson and K. Ramaswamy, "Simple binomial processes as diffusion approximations in financial models," Review of Financial Studies, vol. 3, no. 3, pp. 393-430, 1990.

[5] M. Kounta and M. Lefebvre, "On a discrete version of the CIR process," Journal of Difference Equations and Applications, 2012.

[6] M. Lefebvre and M. Kounta, "Hitting problems for Markov chains that converge to a geometric Brownian motion," ISRN
Discrete Mathematics, vol. 2011, Article ID 346503, 15 pages, 2011.

[7] M. Lefebvre, Applied Stochastic Processes, Springer, NY, USA, 2007.

[8] D. W. Stroock and S. R. Varadhan, Multidimensional Diffusion Processes, Springer, Berlin, Germany, 1979.

[9] M. Abramowitz and I. A. Stegun, Handbook of Mathematical Functions with Formulas, Graphs, and Mathematical Tables, Dover Publications, NY, USA, 1965.

[10] P. M. Batchelder, An Introduction to linear Difference Equations, Dover Publications, Inc., NY, USA, 1967.

[11] A. Bick, "Quadratic-variation-based dynamic strategies," Management Science, vol. 41, no. 4, pp. 722-732, 1995.

[12] H. Geman and M. Yor, "Bessel Processes, Asian Options, and Perpetuities," Mathematical Finance, vol. 3, no. 4, pp. 349-375, 1993.

[13] M. Lefebvre, "Using a lognormal diffusion process to forecast river flows," Water Resources Research, vol. 38, no. 6, pp. 121-128, 2002. 


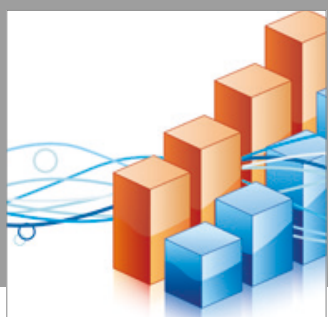

Advances in

Operations Research

vatersals

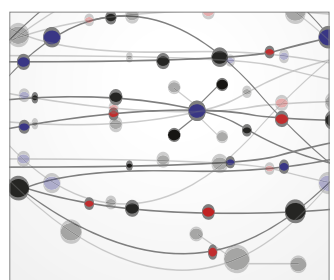

\section{The Scientific} World Journal
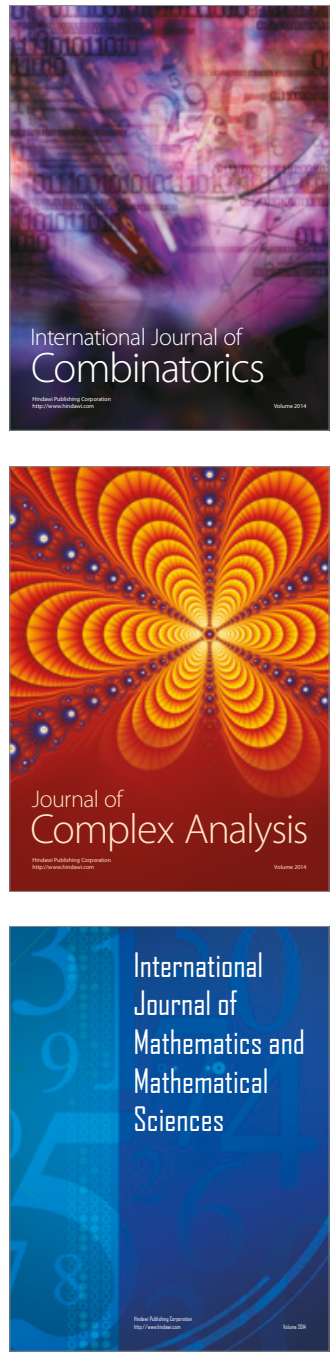
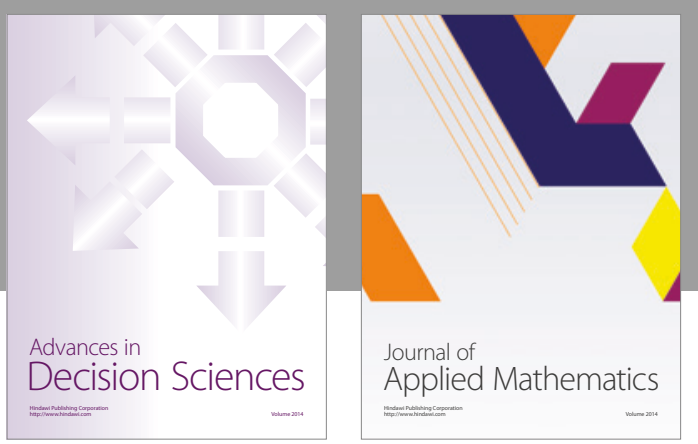

Algebra

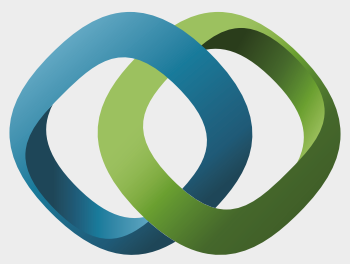

\section{Hindawi}

Submit your manuscripts at

https://www.hindawi.com
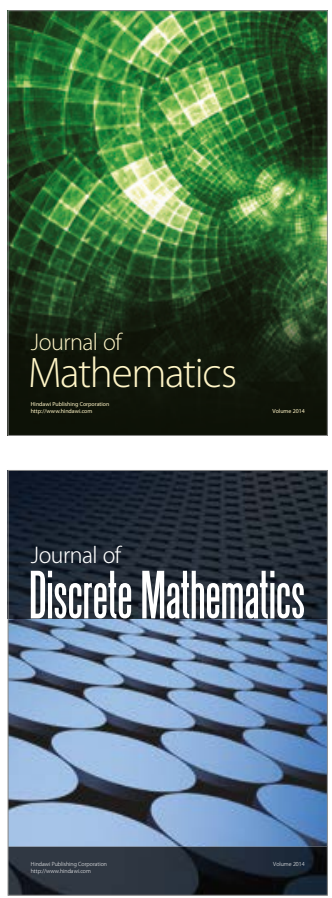

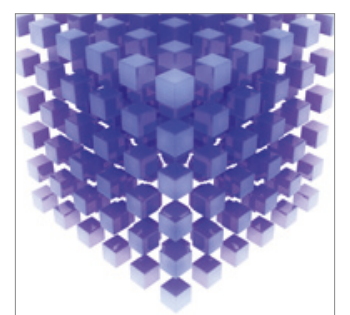

Mathematical Problems in Engineering
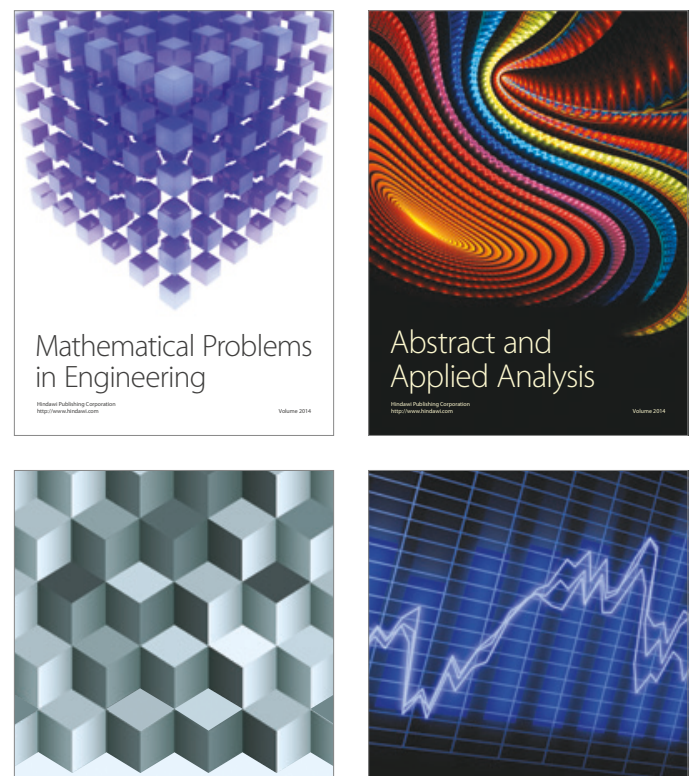

Journal of

Function Spaces

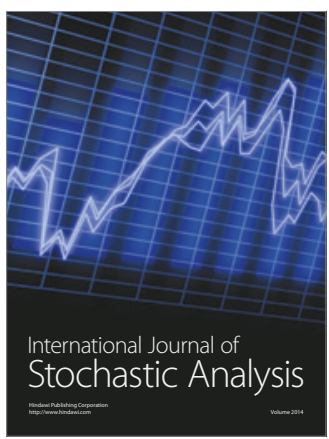

Probability and Statistics
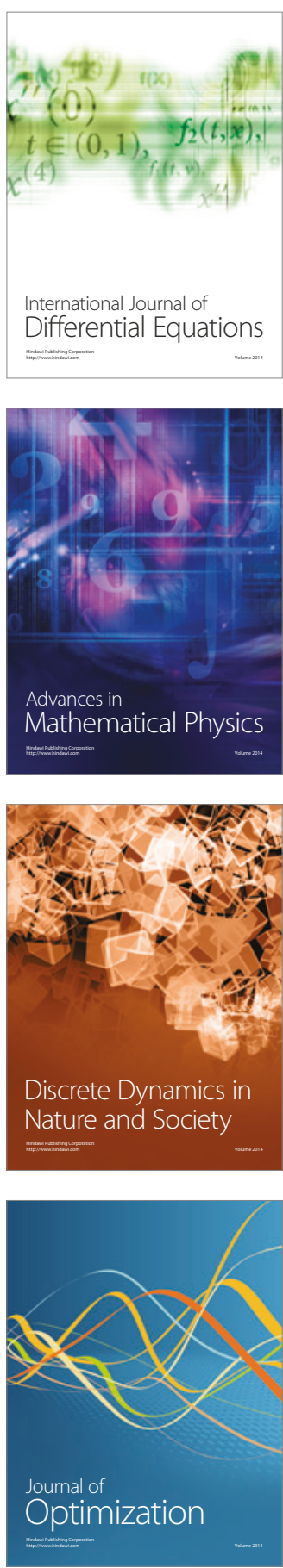